\title{
SOME INEQUALITIES FOR POLYNOMIALS AND THEIR ZEROS
}

\section{ZALMAN RUBINSTEIN}

This note is divided into two parts. In the first part we use some results from the theory of schlicht functions to obtain inequalities involving polynomials and their zeros. Also a new proof is given to a result much used in the theory of polynomials. The second part contains some estimates for the location of the zeros of linear combinations of polynomials. A result due to Biernacki is sharpened and generalized.

I. Lemma. Let

$$
f(z)=\frac{z}{\prod_{k=1}^{n}\left(1-e_{k} z\right)^{m}} .
$$

Denote $\epsilon=\max _{1 \leq k \leq n}\left|\epsilon_{k}\right|$, then

(a) If $\epsilon \leqq 1$ and $m \leqq 2 / n$, the function $f(z)$ is regular and schlicht in the unit disc.

(b) If $m=1, f(z)$ is regular and schlicht in the disc $|z|<1 /(n-1) \epsilon$. In general this result cannot be improved.

Proof. For $|z|=r$, we obtain:

$$
\begin{aligned}
\frac{\partial \arg f(z)}{\partial z} & =\operatorname{Re}\left[z\left(\frac{1}{z}+m \sum_{k=1}^{n} \frac{\epsilon_{k}}{1-\epsilon_{k} z}\right)\right] \\
& =1-\frac{n m}{2}+\frac{m}{2} \sum_{k=1}^{n} \operatorname{Re}\left(\frac{1+\epsilon_{k} z}{1-\epsilon_{k} z}\right) \\
& =1-\frac{n m}{2}+\frac{m}{2} \sum_{k=1}^{n} \frac{1-\left|\epsilon_{k}\right|^{2} r^{2}}{\left|1-\epsilon_{k} z\right|^{2}} .
\end{aligned}
$$

Now $\partial \arg f(z) / \partial z>0$ if $\left|\epsilon_{k}\right| \leqq 1$ and $1-n m / 2 \geqq 0$, for all $0<r<1$, which proves (a) since $\Delta$ arg $f(z)=2 \pi$, when $z$ describes a circle of radius $r$ in the politive direction. Similarly for $m=1$,

$$
\partial \arg f(z) / \partial z>0 \quad \text { if } \sum_{k=1}^{n} \frac{1-\left|\epsilon_{k}\right|^{2} r^{2}}{\left|1-\epsilon_{k} z\right|^{2}}>n-2 .
$$

Since,

Received by the editors August 23, 1963. 


$$
\min \frac{1-\left|\epsilon_{k}\right|^{2} r^{2}}{\left|1-\epsilon_{k} z\right|^{2}} \leqq \frac{1-\epsilon R}{1+\epsilon R}, \quad \text { for } r \leqq R,
$$

(2) is satisfied if $n(1-\epsilon R) /(1+\epsilon R)>n-2$, or $R<1 / \epsilon(n-1)$, which yields (b). The example of Corollary $1(b)$ provides the final part of the lemma.

TheOREM 1. Let $P(z)=a_{n} z^{n}+\cdots+a_{1} z+a_{0}$ have zeros $\alpha_{k}$, $k=1, \cdots, n,\left|\alpha_{k}\right| \leqq 1$, then $P(z)=a_{n}(z-\alpha)^{n}$, where $|\alpha(z)|<1$, for $|z|<1$, and $\alpha(z)$ is a regular function in $|z|>1$.

Proof. By a reciprocal transformation it is necessary and sufficient to prove

$$
\prod_{k=1}^{n}\left(1-z \alpha_{k}\right)=(1-\alpha z)^{n},
$$

where $|\alpha(z)|<1$ for $|z|<1$.

For $|z|<1$ we define $\alpha(z)$ by equation (3), choosing a single-valued branch of the $n$th root. It is easy to see that $\alpha(z)$ is regular in $|z|<1$. It remains to show that $|\alpha(z)|<1$ in $|z|<1$.

By the lemma, $f(z)=z /(1-\alpha z)^{2}$ is regular and schlicht in the unit disc. Since it is also normalized by the conditions $f(0)=0, f^{\prime}(0)=1$, it follows by a well-known estimate [2], that

$$
\frac{|z|}{(1+|z|)^{2}} \leqq \frac{|z|}{|1-\alpha z|^{2}} \leqq \frac{|z|}{(1-|z|)^{2}} .
$$

It follows from (4) that $|\alpha(z)|<3$ in $|z|<1$.

Now since $1 /(1-\alpha z)$ is regular in the disc $|z|<1$, and the series

$$
\frac{1}{1-\alpha z}=1+\alpha z+(\alpha z)^{2}+\cdots
$$

converges for $|z|<1 / 3$, it follows that the series (5) converges for all $z,|z|<1$. Suppose that there is a point $z_{0},\left|z_{0}\right|>1$, such that $\left|\alpha\left(z_{0}\right)\right|>1$, then by the maximum principle we may assume that $\left|z_{0} \alpha\left(z_{0}\right)\right|>1$ and we get a contradiction to the absolute convergence of (5) at $z_{0}$.

TheOREM 2. Let $\prod_{k=1}^{n}\left(1-\alpha_{k} z\right)=(1-\alpha z)^{n}$, where $\left|\alpha_{k}\right| \leqq 1,|\alpha(z)|$ $\leqq 1$, then

$$
\sum_{k=0}^{\infty}\left|\beta_{k}\right|^{2}(2 k+1) \leqq 1
$$


where the numbers $\beta_{k}$ are the coefficients of the expansion in a Taylor series of $\alpha(z)$ in $|z|<1$.

Proof. Since $f(z)=z /(1-\alpha z)^{2}$ is regular, schlicht and normalized in $|z|<1$ the same is true for the function $f_{2}(z)=\left(f\left(z^{2}\right)\right)^{1 / 2}$. Define $F(z)=1 / f_{2}(1 / z)$, then $F(z)$ is schlicht in $|z|>1$ and has the expansion

$$
F(z)=z-\frac{1}{z} \beta_{0}-\frac{1}{z^{3}} \beta_{1}-\cdots .
$$

The theorem follows now by the area theorem for schlicht functions.

The following corollary is deduced easily from the theorems proved.

CoRollary 1. Let $P(z)=\prod_{k=1}^{n}\left(1-\alpha_{k} z\right),\left|\alpha_{k}\right| \leqq 1 /(n-1)$, then

(a) $(1+|z|)^{2} \geqq|P(z)| \geqq(1-|z|)^{2}$ for $|z| \leqq 1$.

(b) $P(z)-z P^{\prime}(z) \neq 0$ for $|z|<1$, and this result is the best possible as the example $P(z)=(1+z /(n-1))^{n}$ shows.

(c) If $c$ is such that $P(z)-z / c \neq 0$ in $|z| \leqq 1$, then

$$
(1+|z|)^{2} \geqq\left|P(z)-\frac{z}{c}\right| \geqq(1-|z|)^{2}
$$

in $|z| \leqq 1$.

(a), (b) follow by the second part of the lemma. (c) follows from the fact that the function $c f(z)(c-f(z))$ is schlicht and regular in $|z|<1$, if $f(z)=z / P(z)$.

II. Lemma. All the zeros of the polynomial

$$
\left(z+e^{i \theta}\right)^{n}-1-n z-\cdots-\left(\begin{array}{c}
n \\
p-1
\end{array}\right) z^{p-1}
$$

are in the disc $|z| \leqq p+1$ for $1 \leqq p<n-1 ; 0 \leqq \theta \leqq 2 \pi$.

Proof. We use the inequality

(6) $1+\left(\begin{array}{c}m+q \\ 1\end{array}\right)(q+1)+\cdots+\left(\begin{array}{c}m+q \\ q-1\end{array}\right)(q+1)^{q-1}<q^{m+q}$.

For $m \geqq 3, q \geqq 2$, (6) was proved by Biernacki [1]. We prove (6) for $m=2, q \geqq 2$ and also indicate the proof for the other cases.

Let $m \geqq 2$. It is easy to verify that

$$
1+\left(\begin{array}{c}
m+q \\
1
\end{array}\right) x+\cdots+\left(\begin{array}{c}
m+q \\
q-1
\end{array}\right) x^{q-1}<\left(\begin{array}{c}
m+q \\
q-1
\end{array}\right)(1+x)^{q-1}
$$

for all $x>0$. 
Substituting $x=q+1$ in (7), one deduces that (6) is true provided

$$
w(m, q)=\left(\begin{array}{c}
m+q \\
q-1
\end{array}\right)(q+2)^{q-1} q^{-(m+q)}<1 .
$$

Since $w(m, q) / w(m+1, q)>1$ for $q>1$, it is enough to consider the case $m=2$. In this case after obvious transformations the equivalent to (6) is the inequality $(1+2 / q)^{q+2}<1+(1+1 / q)^{q+1}(5 / 2+4 / q)$.

Since $(1+2 / q)^{q}$ is increasing, and $(1+1 / q)^{q+1}$ is decreasing, it is enough to show that $(1+2 / q)^{2} e^{2}<1+e(5 / 2+4 / q)$.

Set $x=1+2 / p$, we get $e^{2} x^{2}-2 e x<1+e / 2$, hence

$$
x<(1 / e)\left[1+(2+e / 2)^{1 / 2}\right] \text { and } p>2 e\left[1+(2+e / 2)^{1 / 2}-e\right]^{-1} \text {. }
$$

The remaining cases $(p<50)$ can be verified directly.

The lemma follows now by applying Rouche's theorem, using (6). It follows easily that on the circle $|z|=p+1$,

$$
\left|z+e^{i \theta}\right| n>p^{n}>\left|1+n z+\cdots+\left(\begin{array}{c}
n \\
p-1
\end{array}\right)^{z^{j-1}}\right| .
$$

It can be shown that the lemma is not true for $p=n-1$. We prove now

THEOREM 3. If $P(z)=a_{n} z^{n}+\cdots+a_{0} \neq 0$ in $|z|<1$, then the polynomial $P^{*}(z)=P(z)+\epsilon_{n} a_{n} z^{n}+\epsilon_{n-1} a_{n-1} z^{n-1}+\cdots+\epsilon_{n-p+1} z^{n-p+1} \neq 0$ in $|z|<1 /(p+1)$, for $\left|\epsilon_{k}\right| \leqq 1, k=n-p+1, \cdots, n ; 1 \leqq p<n-1$.

Proof. By a result due to Rahman [3], $P^{*}(z) \neq 0$ in $|z|<1 / t$, where $t$ is the positive root of the equation

$$
(t-1)^{n}=1+n t+\cdots+\left(\begin{array}{c}
n \\
p-1
\end{array}\right) t^{p-1}
$$

By the lemma, with $\theta=\pi, k=p+1, t \leqq p+1$, hence $1 / t \geqq 1 /(p+1)$. Theorem 3 generalizes a result due to Biernacki [1], obtained for $\epsilon_{n}=\epsilon_{n-1}=\cdots=\epsilon_{n-p+1}=-1$.

\section{BIBLIOGRAPHY}

1. M. Biernacki, Sur les ztros des polynômes, Ann. Univ. Mariae Curie-Skłodowska Sect. A 9 (1955), 81-98.

2. G. M. Goluzin, Geometrische Funktionentheorie, VEB Deutscher Verlag der Wissenschaften, Berlin, 1957.

3. Q. I. Rahman, The influence of coefficients on the zeros of polynomials, J. London Math. Soc. 36 (1961), 57-64.

HARVARD UNIVERSITY 